\title{
Las provincias biogeográficas del Componente Mexicano de Montaña desde la perspectiva de los mamíferos continentales
}

\section{The provinces of Mexican Mountain Component based on continental mammals distribution}

\author{
Tania Escalante ${ }^{1 *}$, Gerardo Rodríguez ${ }^{2}$ y Juan J. Morrone ${ }^{3}$ \\ ${ }^{1}$ Laboratorio de Sistemas de Información Geográfica, Departamento de Zoología, Instituto de Biología, UNAM, Apdo. postal 70-153, 04510 México, D. F., \\ México. \\ *Correspondencia: tania_escalante@correo.unam.mx \\ ${ }^{2}$ Laboratorio de Macroecología, Departamento de Ecología de la Biodiversidad, Instituto de Ecología, UNAM, Apdo. postal 70-275, 04510 México, D. F., \\ México. \\ ${ }^{3}$ Museo de Zoología "Alfonso L. Herrera”, Departamento de Biología Evolutiva, Facultad de Ciencias, UNAM, Apdo. postal 70-399, 04510 México, D. F., \\ México.
}

Resumen. Se han utilizado diferentes enfoques con el propósito de analizar los límites entre las regiones Neártica y Neotropical, como índices biogeográficos, análisis panbiogeográficos y análisis biogeográficos cladísticos. A partir de la propuesta de un Componente Mexicano de Montaña basado en datos de la entomofauna, en este trabajo analizamos las provincias de esta zona aplicando el análisis de parsimonia de endemismos (PAE) a datos de mapas de distribución de mamíferos terrestres, con respecto a las regiones Neártica y Neotropical. Encontramos que la provincia de la Sierra Madre Occidental es la que posee la mayor influencia neártica, mientras que la provincia de Chiapas pertenece a la región Neotropical.

Palabras clave: Zona de Transición Mexicana, biogeografía, provincia, región.

Abstract. Different approaches have been used to analyze the limits between the Nearctic and Neotropical regions, such as biogeographic indices, panbiogeographic analyses and cladistic biogeographic analyses. We examine the proposal of a Mexican Mountain Component that was suggested by a previous study of insects, analyzing the provinces of this zone applying the parsimony analysis of endemicity (PAE) to distributional maps of terrestrial mammals, in relation to the Nearctic and Neotropical regions. We found that the Sierra Madre Occidental province has a greater Nearctic influence, whereas the Chiapas province belongs to the Neotropical region.

Key words: Mexican Transition Zone, biogeography, province, region.

\section{Introducción}

Morrone y Márquez (2003) definieron un "Componente Mexicano de Montaña”, el cual está ubicado sobre los principales sistemas montañosos de México y comprende cinco provincias biogeográficas (Fig. 1, exceptuando Chiapas): (1) Sierra Madre Occidental: en el oeste del país, en parte de los estados de Sonora, Chihuahua, Durango, Sinaloa, Nayarit, Jalisco y Zacatecas; (2) Sierra Madre Oriental: ocupa el este del país, en Coahuila, Nuevo León, Hidalgo, San Luis Potosí, Querétaro, Puebla y Veracruz; (3) Eje Volcánico Transmexicano: en el centro de México, esta provincia cruza por los estados de Guanajuato, Distrito Federal, Jalisco, Estado de México, Michoacán, Puebla, Oaxaca, Tlaxcala y Veracruz; (4) Depresión del Balsas: al sur de la provincia del Eje Volcánico Transmexicano, en el

Recibido: 10 noviembre 2004; aceptado: 22 agosto 2005 centro de México, en los estados de Guerrero, Jalisco, Estado de México, Michoacán, Morelos, Oaxaca y Puebla; y (5) Sierra Madre del Sur: en el centro-sur del país, atravesando Michoacán, Guerrero, Puebla y Oaxaca.

Morrone y Márquez (2003) propusieron que este componente representaba un área natural, habitada por un conjunto de taxones homólogos biogeográficamente, la cual fue identificada a partir de la aplicación del método de la panbiogeografía y el análisis de parsimonia de endemismos, y que corresponde a los patrones de distribución neártico y mesoamericano de montaña (Halffter, 1978, 1987). Para Zunino y Zullini (2003), los patrones de distribución de Halffter indican entidades biogeográficas cuyas fronteras resultan a menudo más atenuadas que las de los corotipos o las de regiones o provincias, y se aplican principalmente al estudio de zonas de transición.

La zona de transición entre las regiones Neártica y Neotropical, ubicada en México (Vivó, 1943; Darlington, 

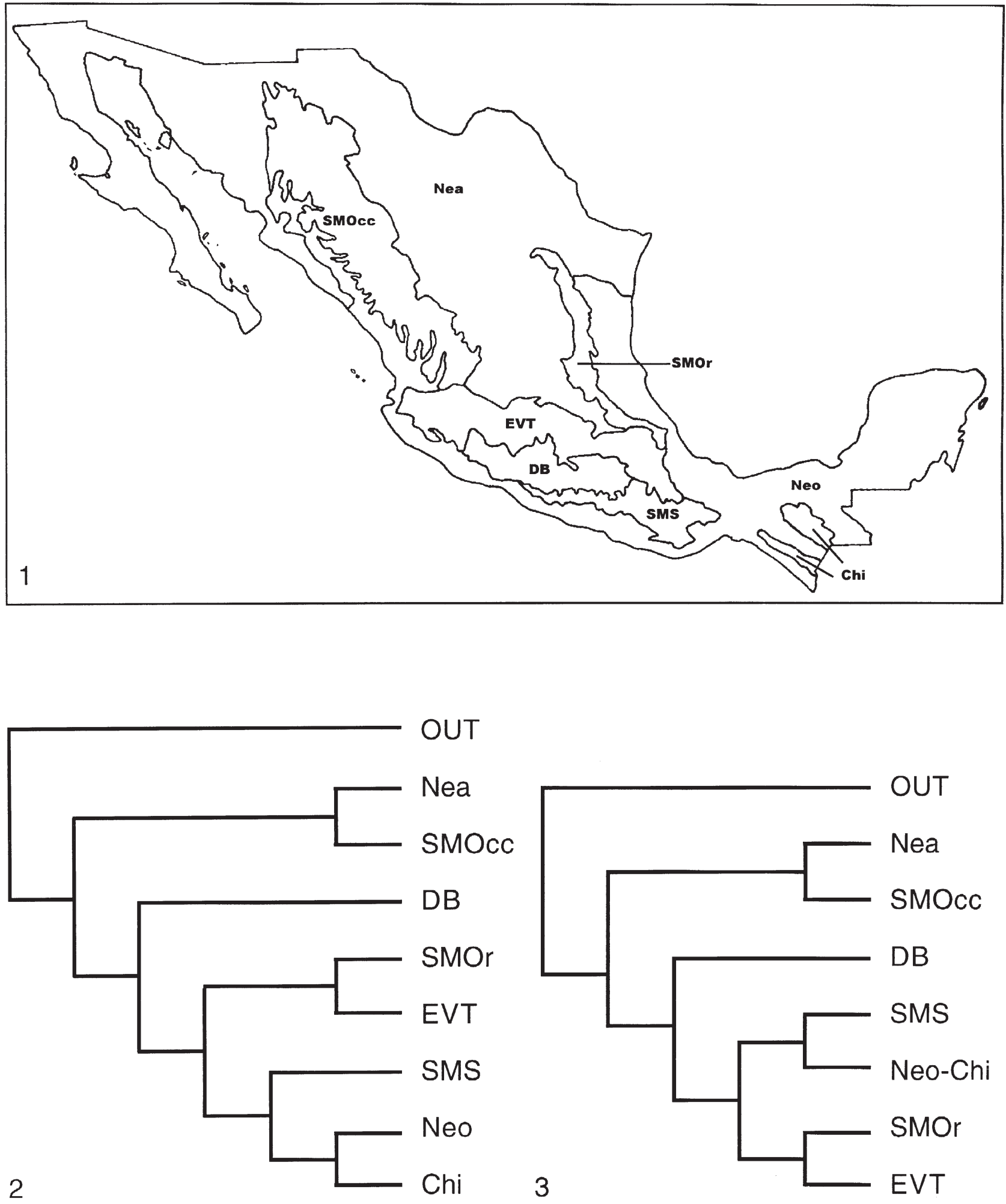

Figuras 1-3. 1, Mapa de las áreas analizadas; 2, cladograma obtenido con la matriz de nueve áreas y 425 especies; 3 , cladograma obtenido con la matriz donde se fusionaron la provincia de Chiapas y la región Neotropical y 425 especies. OUT= área codificada con ceros, Nea= región Neártica, SMOcc= Sierra Madre Occidental, DB= Depresión del Balsas, SMOr= Sierra Madre Oriental, EVT= Eje Volcánico Transmexicano, SMS= Sierra Madre del Sur, Neo= región Neotropical, Chi= Chiapas. 
1957) fue definida formalmente por Halffter (1978). En un sentido amplio, esta “Zona de Transición Mexicana” incluye el sudoeste de los Estados Unidos de América, México y la mayor parte de América Central. Otros autores han utilizado con posterioridad diferentes enfoques para delimitar a esta zona, como índices biogeográficos (Ortega y Arita, 1998), análisis panbiogeográficos (Escalante et al., 2004; Morrone, 2004b) y análisis biogeográficos cladísticos (Marshall y Liebherr, 2000). Escalante et al. (2004) concluyeron que dada la concentración de nodos biogeográficos para mamíferos terrestres neárticos en las provincias del Componente Mexicano de Montaña y Chiapas, en conjunto constituirían la Zona de Transición Mexicana, así como los límites de la región Neártica en México (Fig. 1).

El método del análisis de parsimonia de endemismos o PAE (Rosen, 1988; Rosen y Smith, 1988) permite clasificar áreas de acuerdo con sus taxones compartidos, empleando el criterio de parsimonia (Escalante y Morrone, 2003). El PAE ha sido considerado como una implementación cuantitativa del enfoque panbiogeográfico (Craw et al., 1999; Luna et al., 2000; Morrone y Márquez, 2001; Morrone, 2004a), el cual supone que las barreras geográficas evolucionan junto con las biotas, y su objetivo principal es destacar la importancia de las distribuciones geográficas como objetos directos del análisis (Craw et al., 1999; Morrone, 2004a). Recientemente se ha propuesto una modificación al PAE llamada PAE-PCE (PAE con eliminación progresiva de caracteres, por sus siglas en inglés) (Luna et al., 2000; García-Barros et al., 2002). El PAE-PCE consiste en un procedimiento iterativo donde cada vez que se realiza un análisis de parsimonia y se obtiene un cladograma, las especies que definen clados (sinapomorfias geográficas) son eliminadas y la matriz de datos resultante se analiza nuevamente. El procedimiento termina cuando ya no hay sinapomorfias en los cladogramas. Este método ha sido empleado + para identificar trazos generalizados, puesto que cada nuevo análisis permite obtener nuevos trazos (Luna et al., 2000). Dado que un área de endemismo equivale a un trazo generalizado (Morrone, 2001), el PAE-PCE permitirá en este caso explorar la existencia de relaciones alternativas entre las áreas, cuando se eliminen las sinapomorfias que justifican las relaciones del cladograma anterior.

Los mamíferos conforman un grupo monofilético, cuyas áreas de distribución se encuentran relativamente bien conocidas (v. gr. Hall, 1981; Wilson y Reeder, 1993; Arita y Rodríguez, 2004). Anteriormente hemos analizado los patrones de distribución geográfica que exhiben los mamíferos de México, con base en datos puntuales de registro de ejemplares almacenados en colecciones biológicas y de literatura (Escalante et al., 2002, 2003; Morrone y Escalante, 2002). A pesar de que los datos puntuales han revelado tendencias y patrones importantes, en ocasiones subestiman las áreas de ocupación de los taxones, lo cual puede observarse en cladogramas de PAE poco resueltos (Morrone y Escalante, 2002; Rojas-Soto et al., 2003). Una alternativa es emplear mapas de áreas de distribución generados por especialistas, los cuales contienen las localidades de registro puntuales, pero han sido generalizadas a áreas donde se presume que puede encontrarse al taxón.

En este trabajo, usamos datos de presencia-ausencia obtenidos a partir de mapas de áreas de distribución de los mamíferos terrestres de México, para analizar la relación de las provincias bióticas que se han postulado como pertenecientes al Componente Mexicano de Montaña o Zona de Transición Mexicana, respecto de las regiones Neártica y Neotropical: la Sierra Madre Occidental, la Sierra Madre Oriental, el Eje Volcánico Transmexicano, la Depresión del Balsas y la Sierra Madre del Sur. Puesto que existe controversia sobre la posibilidad de que la provincia de Chiapas pertenezca a la Zona de Transición Mexicana o a la región Neotropical, desarrollamos un segundo análisis fusionando Chiapas con la región Neotropical, con la finalidad de evaluar las relaciones biogeográficas de esta provincia e incluirla o no dentro de la Zona de Transición Mexicana. Por otro lado, también evaluamos el desempeño de la generalización de los mapas de distribución utilizados en el PAE.

\section{Material y métodos}

Se utilizó como referencia la lista de especies de Villa y Cervantes (2003). De las 449 especies de mamíferos terrestres de México, se eliminaron 24 que se distribuyen únicamente en islas, usándose los datos de distribución de las 425 especies restantes. Los mapas de distribución utilizados fueron tomados de Patterson et al. (2003), Costello y Rosenberg (2004) e InfoNatura (2004), los cuales se superpusieron a un mapa de América en el Sistema de Información Geográfica ArcView 3.2 (ESRI, 1999). El primer análisis de parsimonia se llevó a cabo con una matriz de 425 especies y nueve áreas: un área raíz (codificada con ceros), la región Neártica, la región Neotropical, la Sierra Madre Occidental, la Sierra Madre Oriental, el Eje Volcánico Transmexicano, la Depresión del Balsas, la Sierra Madre del Sur y Chiapas (Fig. 1). Si la especie se encontraba distribuida en un área con más del $10 \%$ de su distribución superpuesta al área se codificaba con " 1 ", si la superposición era menor a este porcentaje se codificaba con “?”, y si no se distribuía en el área se codificaba con "0". Con la finalidad de comparar el efecto de la generalización de los mapas de distribución empleados sobre los cladogramas del PAE, se elaboró una segunda matriz donde se remplazaron los "?" por " 0 ". Se aplicó una búsqueda heurística (Multiple TBR+TBR) en WinClada (Nixon, 1999) y Nona (Goloboff, 1993) a ambas matrices, eliminando los taxones no informativos.

Una vez que se obtuvo el cladograma del primer análisis, se aplicó la técnica de PAE-PCE de García-Barros et al. (2002). Para ello, se identificaron las especies que definieron los clados, se eliminaron de la matriz, se realizó otra búsqueda heurística, y se repitió el procedimiento hasta no 
obtener sinapomorfias en los cladogramas generados.

Adicionalmente se realizaron dos análisis fusionando la provincia de Chiapas con la región Neotropical, para explorar si su relación con la región Neotropical influye en los resultados.

\section{Resultados}

En el primer análisis de la matriz con “?”, "0" y " 1 ” se desactivaron 208 especies no informativas, y se obtuvo un único cladograma con 365 pasos, índice de consistencia de 0.59 e índice de retención de 0.60 (Fig. 2). De las especies no informativas, se identificaron 38 exclusivas de las seis provincias. Para la matriz codificada sólo con "0" y "1", las especies no informativas desactivadas fueron 189, y también se obtuvo un único cladograma con 425 pasos, índice de consistencia de 0.55 e índice de retención de 0.55 , el cual muestra las mismas relaciones que el de la matriz con "?" (Fig. 2). Dado que ambos cladogramas presentaron las mismas relaciones, es decir, la generalización de los mapas de distribución usados no mostró influencia importante en el PAE, el PAE-PCE se llevó a cabo de manera secuencial sólo para esta matriz, cuyos resultados se muestran en el Cuadro 1.

La matriz con "?", " 0 " y "1" donde se fusionaron la provincia de Chiapas y la región Neotropical tuvo 255 especies no informativas. A partir de ella se obtuvo un único cladograma con 290 pasos, índice de consistencia de 0.58 e índice de retención de 0.54 (Fig. 3). La matriz con " 0 " y " 1 " con estas áreas fusionadas tuvo 235 especies no informativas, a partir de ellas se obtuvo un solo cladograma con 342 pasos, índice de consistencia de 0.55 e índice de retención de 0.51 , con la misma topología que el anterior.

\section{Discusión y conclusiones}

Una zona de transición es un área donde dos o más biotas que habían evolucionado en condiciones de independencia recíproca entran en contacto, superponiéndose y mezclándose en distintas medidas, simétrica o asimétricamente (Zunino y Zullini, 2003). Morrone (2004b) mencionó que las zonas de transición, localizadas en los límites entre regiones biogeográficas, representan eventos de hibridación biótica, promovidos por cambios ecológicos e históricos que permiten la mezcla de componentes bióticos diferentes.

El Componente Mexicano de Montaña es equivalente a la Zona de Transición Mexicana, un área que exhibe una alta diversidad, lo cual es resultado de importantes procesos de especiación que se llevaron a cabo cuando hibridizaron las regiones Neártica y Neotropical. Los mamíferos de México exhiben una alta riqueza de especies exclusivas de las seis provincias analizadas (38 taxones): (1) Sierra Madre Occidental: Neotoma palatina, Peromyscus polius, Spermophilus madrensis y Tamias bulleri; (2) Sierra Madre Oriental: Cratogeomys neglectus, Neotoma angustapalata, Perognathus merriami, Peromyscus furvus, Peromyscus ochraventer y Sciurus hallen; (3) Eje Volcánico Transmexicano: Cratogeomys gymnurus, Cratogeomys merriami, Cratogeomys tylorhinus, Cratogeomys zinseri, Nelsonia goldmani, Neotoma nelsoni, Neotomodon alstoni, Orthogeomys lanius, Pappogeomys alcorni, Pappogeomys bulleri, Peromyscus mekisturus, Reithrodontomys chrysopsis, Reithrodontomys hirsutus, Romerolagus diazi, Spermophilus perotensis y Zygogeomys trichopus; (4) Depresión del Balsas: Spermophilus adocetus; (5) Sierra Madre del Sur: Cryptotis goldmani, Microtus umbrosus, Oryzomys caudatus, Peromyscus megalops y Sylvilagus insonus; y (6) Chiapas: Heteromys nelsoni, Peromyscus zarhynchus, Sorex sclateri, Sorex stizodon, Tylomys bullaris y Tylomys tumbalensis.

En los cladogramas, la provincia de la Sierra Madre Occidental resultó estrechamente relacionada con la región Neártica, con 14 sinapomorfias geográficas; mientras que la provincia de Chiapas lo fue con la región Neotropical, con 37 sinapomorfias. Estos resultados son congruentes con la existencia de la Zona de Transición Mexicana, donde la

Cuadro 1. Análisis de parsimonia de endemismos con eliminación progresiva de caracteres, para la matriz codificada sólo con 0 y 1.

\begin{tabular}{llllll}
\hline $\begin{array}{l}\text { Análisis } \\
\text { secuencial }\end{array}$ & $\begin{array}{l}\text { Número de } \\
\text { especies } \\
\text { desactivadas }\end{array}$ & $\begin{array}{l}\text { Número de } \\
\text { cladogramas }\end{array}$ & $\begin{array}{l}\text { Número de } \\
\text { pasos, CI y RI }\end{array}$ & $\begin{array}{l}\text { Relación de la región } \\
\text { Neártica con las } \\
\text { provincias }\end{array}$ & $\begin{array}{l}\text { Relación de la región } \\
\text { Neotropical con las provincias }\end{array}$ \\
\hline 1 & 189 & 1 & $425,0.55,0.55$ & Sierra Madre Occidental & Chiapas \\
2 & 98 & 1 & $251,0.54,0.55$ & Sierra Madre Oriental & Chiapas \\
3 & 67 & 1 & $137,0.51,0.42$ & En la base del cladograma & Chiapas \\
4 & 28 & 7 & $80,0.53,0.45$ & Sierra Madre Oriental & Diferentes provincias \\
5 & 26 & 2 & $27,0.62,0.61$ & Sierra Madre Oriental & Depresión del Balsas \\
6 & 10 & 6 & $12,0.58,0.58$ & $\begin{array}{l}\text { Sierra Madre Occidental y } \\
\text { región Neotropical }\end{array}$ & $\begin{array}{l}\text { Sierra Madre Occidental y } \\
\text { región Neártica }\end{array}$ \\
7 & 4 & 1 & $3,1,1$ & Neotropical & Neártica \\
\hline
\end{tabular}


Sierra Madre Occidental poseería mayor influencia neártica y la de Chiapas mayor influencia neotropical.

No existen muchos trabajos que consideren los patrones de distribución geográfica de los mamíferos para delimitar la zona de transición en México. Para Fa y Morales (1998), la demarcación de las regiones Neártica y Neotropical está asociada a factores climáticos y de hábitat; estos autores trazaron una línea que separa las regiones templadas de las tropicales aproximadamente al centro del país. Ortega y Arita (1998) distinguieron una zona transicional basada en patrones de distribución de murciélagos y un índice biogeográfico, la cual está definida básicamente por la Sierra Madre Occidental, la Sierra Madre Oriental, el Eje Volcánico Transmexicano, el norte de la Cuenca del Río Balsas y las tierras altas de Oaxaca y Chiapas. Morrone (2004b) incorporó los resultados de análisis panbiogeográficos con diversos taxones, y restringió la Zona de Transición Mexicana en sentido estricto al Componente Mexicano de Montaña, el cual posee cinco provincias: la Sierra Madre Occidental, la Sierra Madre Oriental, el Eje Volcánico Transmexicano, la Cuenca del Balsas y la Sierra Madre del Sur. Escalante et al. (2004), en su estudio panbiogeográfico con 28 especies de mamíferos neárticos, no consideraron a la Cuenca del Balsas dentro de la Zona de Transición Mexicana, ya que no encontraron nodos biogeográficos en ella, pero incorporaron a la provincia de Chiapas, la cual posee biota neártica relictual.

En nuestros análisis, la provincia de Chiapas se mantuvo como el área hermana de la región Neotropical. Cuando fusionamos ambas, mostraron 41 apomorfías geográficas (Fig. 3), por lo que suponemos existe una relación muy estrecha entre ellas, y probablemente Chiapas pertenezca a la región Neotropical. Aunque Chiapas presenta taxones relictuales de origen neártico (Escalante et al., 2004), hay mayor evidencia para que sea incluida en la región Neotropical mas que en la Zona de Transición Mexicana. Sugerimos que esta provincia sea analizada más detalladamente con otros taxones, para establecer su pertenencia a la zona de transición o a la región Neotropical.

Es relevante mencionar que la Zona de Transición Mexicana se comportó como un área parafilética, donde las regiones Neártica y Neotropical se insertan dentro de ella. En este sentido, quizá no sería adecuado utilizar el término "componente". También cabe mencionar la posibilidad de que las regiones Neártica y Neotropical tampoco sean monofiléticas, lo cual deberá ser probado posteriormente.

Actualmente hay controversia acerca del uso de mapas de distribución como los que se emplean en este trabajo. Es necesario tener en cuenta que los mapas de distribución son generalizaciones de las distribuciones geográficas, las que en muchos casos pueden caer en sobrestimaciones. En este caso, el efecto que tuvo la generalización de los mapas utilizados sobre los cladogramas del PAE no fue muy importante, ya que aunque no había certeza de que en ciertos casos las especies estuvieran o no presentes en un área (codificación con “?”, donde había menos del 10\% de superposición del mapa de distribución en el área), las relaciones entre las áreas no cambiaron respecto a las matrices donde se suponía que no estaba el taxón (codificación con " 0 "). Por el contrario, el uso de datos puntuales de localidades donde se han registrado ejemplares en ocasiones puede subestimar las áreas de distribución. Una posibilidad intermedia entre estas consiste en emplear modelos de nicho ecológico proyectados como la distribución geográfica generados mediante herramientas como los algoritmos genéticos (GARP, Stockwell y Noble, 1992; Stockwell, 1999). Sería conveniente realizar este mismo estudio empleando estos modelos, los cuales ya se han comenzado a utilizar con propósitos biogeográficos (Escalante et al., 2003; Rojas-Soto et al., 2003).

Este trabajo, así como los realizados por Morrone y Márquez (2001, 2003), constituyen un análisis de homología biogeográfica primaria o el estadio de generación de hipótesis de la historia biogeográfica de México, obtenidos mediante análisis panbiogeográficos y PAE (Morrone, 2001; 2004a). Más adelante, será necesario contrastar la hipótesis propuesta (homología biogeográfica secundaria) mediante un análisis biogeográfico cladístico (Morrone, 2001, 2004a).

\section{Agradecimientos}

T. Escalante agradece la beca posdoctoral de DGAPAUNAM 2004-2005. J. J. Morrone agradece el apoyo económico del proyecto CONACYT 36488. David EspinosaOrganista leyó críticamente el manuscrito preliminar.

\section{Literatura citada}

Arita, H. T. y G. Rodríguez. 2004. Patrones geográficos de diversidad de los mamíferos terrestres de América del Norte. Instituto de Ecología, UNAM. Base de datos SNIB-Conabio proyecto Q068. México, D.F. http:// www.conabio.gob.mx/informacion/mamiferos/doctos/ presentacion.html

Costello, R. y A. Rosenberg. 2004. North American Mammals. Smithsonian Institution, National Museum of Natural History. http://www.mnh2.si.edu/education/mna

Craw, R. C., J. R. Grehan, y M. J. Heads. 1999. Panbiogeography: tracking the history of life. Oxford Biogeography Series 11. Oxford University Press, Nueva York. 229 p.

Darlington, P. J. 1957. Zoogeography: The geographical distribution of animals. Wiley, New York. 675 p.

Escalante, T., D. Espinosa y J. J. Morrone. 2002. Patrones de distribución geográfica de los mamíferos terrestres de México. Acta Zoológica Mexicana (n. s.) 87:47-65.

Escalante, T., D. Espinosa y J. J. Morrone. 2003. Using 
Parsimony Analysis of Endemicity to analyze the distribution of Mexican land mammals. Southwestern Naturalist 48:563-578.

Escalante, T. y J. J. Morrone. 2003. ¿Para qué sirve el Análisis de Parsimonia de Endemismos?. In Una perspectiva latinoamericana de la biogeografía, J. J.Morrone y J. Llorente (eds.). Las Prensas de Ciencias, UNAM, México, D.F. p. 167-172.

Escalante, T., G. Rodríguez y J. J. Morrone. 2004. The diversification of Nearctic mammals in the Mexican Transition Zone. Biological Journal of the Linnean Society 83: 327-339.

ESRI. 1999. ArcView GIS 3.2. Environmental Systems Research Institute, Inc. New York.

Fa, J. E. y L. M. Morales. 1998. Patrones de diversidad de mamíferos de México. In Diversidad biológica de México: orígenes y distribución, T. P. Ramamoorthy, R. Bye, A. Lot y J. Fa (eds.). Instituto de Biología, UNAM., México, D. F. p. 315-352.

García-Barros, E., P. Guerrea, M. Luciañez, J. Cano, M. Munguira, J, Moreno, H. Sainz, M. Sanz y J. C. Simón. 2002. Parsimony analysis of endemicity and its application to animal and plant geographical distributions in the Ibero-Balearic region (western Mediterranean). Journal of Biogeography 29:109-124.

Goloboff, P. 1993. Nona. Cladistics 9:83-91.

Halffter, G. 1978. Un nuevo patrón de dispersión en la Zona de Transición Mexicana: El mesoamericano de montaña. Folia Entomologica Mexicana 39-40:219-222.

Halffter, G. 1987. Biogeography of the montane entomofauna of Mexico and Central America. Annual Review of Entomology 32:95-114.

Hall, E. R. 1981. The mammals of North America, vols. I y II, John Wiley, New York. 1181 p.

InfoNatura. 2004. InfoNatura: Birds, mammals, and amphibians of Latin America. Version 3.1. Arlington, Virginia. NatureServe. http://www.natureserve.org/ infonatura. (Acceso: mayo 4, 2004 ).

Luna, I., O. Alcántara, J. J. Morrone y D. Espinosa. 2000. Track analysis and conservation priorities in the cloud forests of Hidalgo, Mexico. Diversity and Distributions 6:137-143.

Marshall, C. J. y J. K. Liebherr. 2000. Cladistic biogeography of the Mexican transition zone. Journal of Biogeography 27:203-216.

Morrone, J. J. 2001. Homology, biogeography and areas of endemism. Diversity and Distributions 7:297-300.

Morrone, J. J. 2004a. Homología biogeográfica: Las coordenadas espaciales de la vida. Cuadernos 37, Instituto de Biología, UNAM, México, D. F. 199 p.

Morrone, J. J. 2004b. Panbiogeografía, componentes bióticos y zonas de transición. Revista Brasileira de Entomologia 48:149-162.

Morrone J. J. y T. Escalante. 2002. Parsimony Analysis of Endemicity (PAE) of Mexican terrestrial mammals at different area units: when size matters. Journal of Biogeography 29:1095-1104.

Morrone J. J. y J. Márquez. 2001. Halffter's Mexican Transition Zone, beetle generalized tracks, and geographical homology. Journal of Biogeography 28: 635-650.

Morrone, J. J. y J. Márquez. 2003. Aproximación a un atlas biogeográfico mexicano: Componentes bióticos principales y provincias biogeográficas. In Una perspectiva latinoamericana de la biogeografía, J. J. Morrone y J. Llorente (eds.). Las Prensas de Ciencias, UNAM, México, D.F. p. 1-4.

Nixon, K.C. 1999. Winclada (beta) ver. 0.9.9. Publicado por el autor, Ithaca, Nueva York.

Ortega, J. y H. T. Arita. 1998. Neotropical-Nearctic limits in Middle America as determined by distributions of bats. Journal of Mammalogy 79:772-783.

Patterson, B. D., G. Ceballos, W. Sechrest, M. F. Tognelli, T. Brooks, L. Luna, P. Ortega, I. Salazar y B. E. Young. 2003. Digital Distribution Maps of the Mammals of the Western Hemisphere, version 1.0. NatureServe, Arlington, Virginia.

Rojas-Soto, O. R., O. Alcántara-Ayala y A. G. Navarro. 2003. Regionalization of the avifauna of the Baja California Peninsula, México: A parsimony analysis of endemicity and distributional modeling approach. Journal of Biogeography 30:449-461.

Rosen, B. R. 1988. From fossils to earth history: applied historical biogeography. In Analytical biogeography: an integrated approach to the study of animal and plant distributions, A. A. Myers y P. Giller (eds.). Chapman and Hall, London, p. 437-481.

Rosen, B. R. y A. B. Smith. 1988. Tectonics from fossils? Analysis of reef-coral and sea-urchin distributions from late Cretaceous to Recent, using a new method. In Gondwana and Tethys, M.G. Audley-Charles y A. Hallam (eds.). Special Publication of the Geological Society of London 37, London, p. 275-306.

Stockwell, D. R. 1999. Genetic Algorithms II. In Machine learning methods for ecological applications, A. H. Fielding (ed.). Kluwer Academic Boston, Massachusetts, p. 123-144.

Stockwell, D. R. y I. R. Noble. 1992. Induction of sets of rules from animal distribution data: a robust and informative method of data analysis. Mathematics and Computers in Simulation 32:249-254.

Villa, B. y F. A. Cervantes. 2003. Los mamíferos de México. Instituto de Biología, UNAM- Grupo Editorial Iberoamérica, México, D. F. 140 p.

Vivó, J. A. 1943. Los límites biogeográficos en América y la zona cultural mesoamericana. Revista Geográfica 3:109-131. 
Wilson, D. E. y D. M. Reeder. 1993. Mammal species of the world: a taxonomic and geographic reference. Smithsonian Institution Press - American Society of Mammalogists, Washington, D.C., 1206 p.
Zunino, M. y A. Zullini. 2003. Biogeografía: la dimensión espacial de la evolución. Fondo de Cultura Económica, México, D. F. 359 p. 